\title{
Satisfação sexual na demência
}

\section{Sexual satisfaction in dementia}

\author{
Marcela Moreira Lima Nogueira ${ }^{1}$, Denise Brasil ${ }^{1}$, Maria Fernanda Barroso de Sousa, Raquel Luiza Santos¹, Marcia \\ CRistina Nascimento Dourado ${ }^{1}$
}

1 Instituto de Psiquiatria da Universidade Federal do Rio de Janeiro (IPUB/UFRJ), Rio de Janeiro, RJ, Brasil.

Centro de Doença de Alzheimer e Outros Transtornos Mentais na Velhice do Instituto de Psiquiatria da Universidade Federal do Rio de Janeiro (IPUB/UFRJ).

Recebido: 25/5/2012 - Aceito: 15/2/2013

\begin{abstract}
Resumo
Contexto: A demência pode resultar em comprometimento da intimidade e sexualidade de casais idosos. Objetivos: Avaliar alterações na atividade sexual, bem como os fatores de satisfação e/ou insatisfação sexual de casais nos quais um dos parceiros possua demência. Método: Busca nas bases de dados ISI, PubMed/ Medline e SciELO de artigos sobre sexualidade na demência, entre janeiro de 1990 e março de 2012, utilizando as palavras-chave: "demência", "satisfação sexual", "intimidade" e "sexualidade". Resultados: Foram encontrados 12 artigos. A sobrecarga de cuidados e a alteração de papéis na relação conjugal foram consideradas as principais causas para o declínio da atividade sexual. A disfunção erétil em pacientes e cônjuges, a capacidade decisória para o consentimento da relação sexual por parte do paciente demenciado e os problemas referentes à idade e à saúde (física e emocional) do cônjuge e/ou paciente foram os fatores associados à insatisfação sexual. Conclusão: A intimidade e a atividade sexual dos casais em que um dos parceiros é portador de demência são influenciadas negativamente pela relação de cuidados decorrente da doença e pela sobrecarga dos cônjuges. Por outro lado, a atividade sexual pode ser positivamente substituída por demonstrações de carinho e empatia entre os cônjuges.
\end{abstract}

Nogueira MML, et al. / Rev Psiq Clín. 2013;40(2):77-80

Palavras-chave: Demência, satisfação sexual, sexualidade.

\begin{abstract}
Background: Dementia may result on impairment in intimacy and sexuality of elderly couples. Objectives: Evaluate changes in sexual activity, as well as the factors which cause sexual satisfaction and/or dissatisfaction in couples in which one of the partners has dementia. Method: A search at ISI, PubMed/Medline and SciELO was made for articles about sexuality in dementia, from January 1990 to March 2012, using the keywords: "dementia", "sexual satisfaction", "intimacy" and "sexuality". Results: Twelve articles were selected. The burden of care and the change of roles in couples' relationship were the main reasons for decrease of sexual activity. Erectile dysfunction in patients and spouses, the decision-making capacity for sexual relationship from the patient who has dementia and the problems related to age and health (physical and emotional) of spouses and/or patients were considered as reasons associated with sexual dissatisfaction. Discussion: When one partner has dementia, the couples' intimacy and sexual activity are negatively influenced by the relationship of care related to the disease and by the spouse's burden. On the other hand, sexual activity may be positively replaced by displays of affection and empathy between the couple.
\end{abstract}

Nogueira MML, et al. / Rev Psiq Clín. 2013;40(2):77-80

Keywords: Dementia, sexual satisfaction, sexuality.

\section{Introdução}

Amor, intimidade sexual e sexualidade são características que definem o indivíduo e frequentemente influenciam a forma de viver e envelhecer de cada ser humano ${ }^{1}$. Até recentemente, ainda se acreditava que o declínio da função sexual era inevitável face à menopausa e à instalação progressiva de disfunções da ereção masculina. Atualmente, existem evidências de que interesse e inibição sexual coexistem na população idosa saudável, correlacionados às características culturais e diferenciação intergêneros².

A síndrome demencial é caracterizada pelo comprometimento da memória associado à alteração em uma ou mais funções cognitivas ${ }^{3}$, sendo comum a presença de sintomas neuropsiquiátricos. Os idosos portadores de demência ainda são considerados incapazes de manter atividade sexual normal e ter prazer ${ }^{4}$. Dessa forma, a sexualidade na demência tem sido estudada, principalmente, em relação à presença de comportamento sexual inadequado ${ }^{5}$.

Relatos sobre a atividade sexual na demência sugerem que o cônjuge saudável demonstra constrangimento em manter uma prática com um parceiro que não sabe mais seu nome ou já não é capaz de reconhecê-lo ${ }^{6,7}$. Além disso, cônjuges masculinos descrevem sentimentos de culpa por manter relações sexuais sem o consentimento ou compreensão da esposa, já que, com a progressão da doença, a consciência dos déficits e a capacidade decisória declinam ${ }^{8-10}$. Assim sendo, o dilema central da atividade sexual na demência está relacionado ao conflito entre a proteção de pacientes e cônjuges e a manutenção das necessidades sexuais desses casais ${ }^{11}$.

Essa revisão sistemática tem como objetivo avaliar os artigos sobre a atividade e a satisfação sexual em idosos com demência e seus cônjuges, uma vez que a relação sexual e sua influência na vida íntima do casal também podem ser fatores importantes para o desenvolvimento de sobrecarga no cuidador ${ }^{12}$.

\section{Método}

Foi realizada uma busca nas bases PubMed, Science Citation Index (Institute for Scientific Information - ISI) e na Revista Sexuality and 
Disability, procurando artigos de janeiro de 1990 a março de 2012, em português, inglês, espanhol, italiano e francês, que abordassem a atividade sexual em idosos portadores de demência. Utilizaram-se as palavras-chaves "dementia", "sexual satisfaction", "intimacy" e "sexuality", com as combinações "sexual satisfaction AND dementia", "intimacy AND dementia", e "sexuality AND dementia". Foram adotados os seguintes limites: humans, English, French, Italian, Spanish, Latin, Portuguese, middle aged, 45+ years, 65+ years, 80+ years, publication date from 1990/01/01 to 2012/03/01.

Foram excluídos: artigos sem abstract ou incompletos; revisões sistemáticas e cartas do editor; artigos sobre intervenção com profissionais de saúde e intervenções farmacológica, psicoterápica, musical, legal, linguística e em instituição; artigos com foco na sobrecarga do cuidador e no paciente sem relação com a sexualidade; artigos que abordassem outras patologias, menopausa, disfunção sexual, diferenciação sexual, comportamento sexual inadequado, hipersexualidade, homossexualidade; e avaliação da atividade sexual de casais idosos saudáveis.

Todos os artigos selecionados foram lidos por dois avaliadores e, então, agrupados de forma independente quanto à metodologia, ao desenho de estudo, aos instrumentos utilizados e aos resultados obtidos. Os resumos aprovados pelos dois avaliadores foram incluídos na revisão. Os artigos que motivaram discordância entre os avaliadores foram submetidos a um terceiro parecer. Os estudos selecionados foram examinados para avaliar as alterações na atividade sexual, bem como os fatores de satisfação e/ou insatisfação sexual em idosos portadores de demência e seus cônjuges. O desenho e os instrumentos utilizados foram também levantados.

\section{Resultados}

Foram encontrados 266 artigos no PubMed e 102 na base ISI. Retiradas as referências cruzadas em mais de uma base, foram selecionados nove artigos. Foram adicionados, ainda, três artigos encontrados na Revista Sexuality and Disability (Tabela 1).

\section{Avaliação metodológica dos estudos}

Entre os artigos selecionados, dois eram estudos randomizados ${ }^{12,13}$, nove eram não randomizados ${ }^{6,8,14-20} \mathrm{e}$ um estudo apresentou o grupo de avaliação não randomizado e o grupo controle randomizado ${ }^{21}$. Foram encontrados estudos transversais ${ }^{8,13,15,17-19}$, caso controle ${ }^{12,13}$, de metodologia qualitativa ${ }^{14,16}$, estudo de caso $^{6}$ e intervenção ${ }^{21}$. Escalas específicas para avaliação da atividade sexual foram encontradas em quatro estudos ${ }^{13,18-20}$; entrevistas semiestruturadas foram utilizadas em cinco artigos ${ }^{12,14-16,21}$; e dois artigos utilizaram uma combinação de escalas específicas e entrevistas semiestruturadas ${ }^{8,17}$.

Dez estudos analisaram apenas a visão do cuidador sobre as mudanças na relação conjugal e sexual do casal6,12,14-21, enquanto dois abrangeram os pontos de vista do parceiro sexual e do paciente ${ }^{8,13}$.

Em onze artigos, o cônjuge saudável era do sexo feminino ${ }^{8,12-21}$, exceto um estudo de caso, com cônjuge do sexo masculino ${ }^{6}$.

\section{Fatores de satisfação e/ou insatisfação sexual}

Em quatro artigos, o gênero teve influência direta na satisfação ou insatisfação sexual 6,12,14,18, demonstrando que cônjuges do sexo feminino apresentaram maior desinteresse sexual quando comparadas aos cônjuges do sexo masculino. Em seis estudos, a sobrecarga do cônjuge com os cuidados diários com o paciente demenciado foi apresentada como o principal motivo para a falta ou diminuição da atividade sexual $8,12,14,16,19-20$. Outras causas atribuídas à ausência de relações sexuais foram o medo de rejeição por parte das esposas 6 , a disfunção erétil em pacientes e cônjuges ${ }^{8,19}$, a necessidade de 0 paciente ser capaz de reconhecer seu companheiro ${ }^{6}$, a capacidade decisória para o consentimento da atividade sexual por parte do paciente demenciado $\mathrm{o}^{6,8,14} \mathrm{e}$ as consequências relacionadas à idade $\mathrm{e}$
Tabela 1. Artigos excluídos

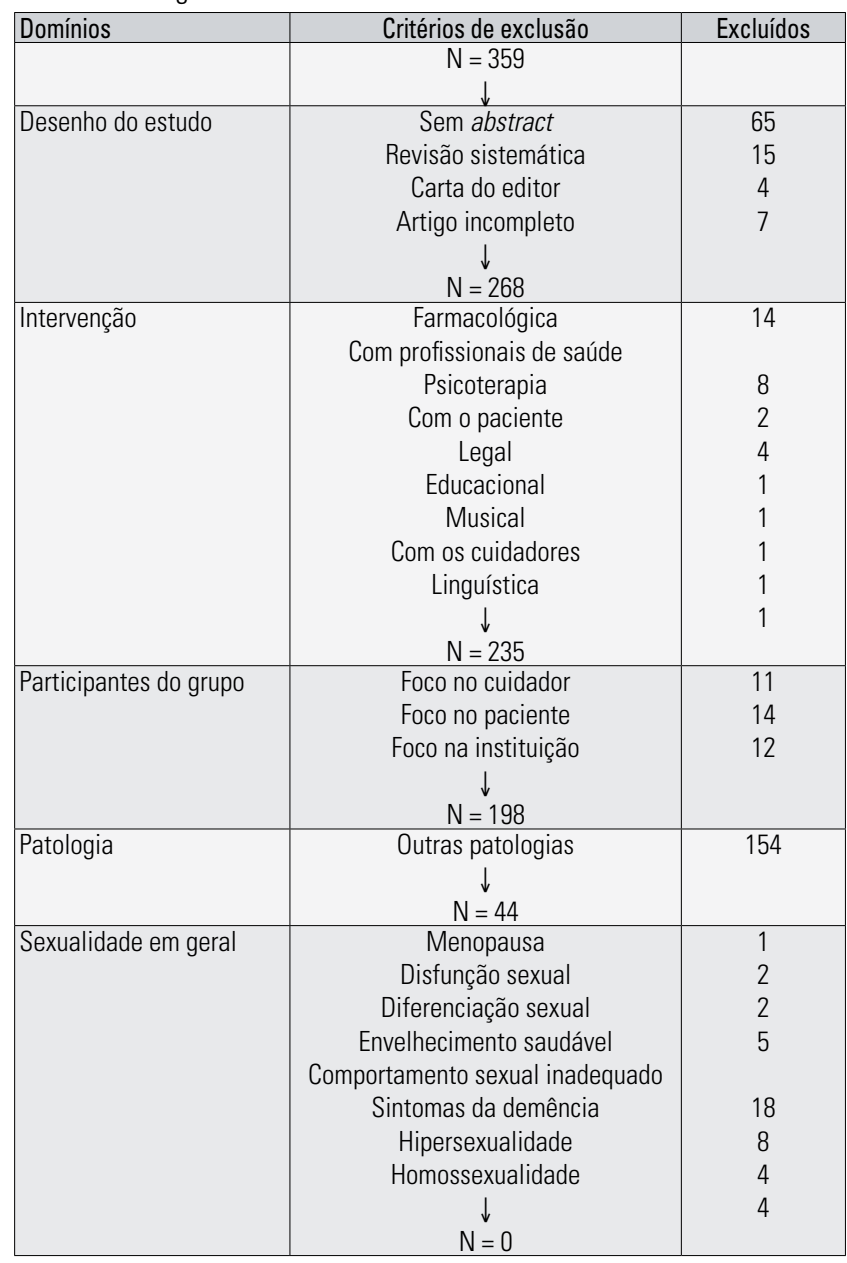

à saúde (física e emocional) do cônjuge e/ou paciente14,19,21. A apatia, a depressão e a falta de interesse pela continuidade da atividade sexual, por parte do paciente, também foram citadas como motivos

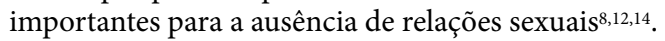

Dois estudos sugerem que a atividade sexual pode ser substituída positivamente por demonstrações de carinho e empatia entre os cônjuges ${ }^{6,19}$ (Tabela 2).

\section{Discussão}

A atividade sexual em casais de idosos nos quais um dos parceiros é portador de demência ainda é pouco estudada. Os artigos selecionados para essa revisão apresentaram diferenças metodológicas significativas (poucos estudos randomizados ${ }^{12,13}$ e ausência de um instrumento que possa ser considerado padrão-ouro para a avaliação $\left.0^{6,8,12-21}\right)$. Dessa forma, a falta de sistematização dos métodos de avaliação dificulta a replicação desses trabalhos, o que coloca em questão a confiabilidade dos resultados obtidos.

Entre os dados analisados, observou-se que a presença da síndrome demencial causa declínio na intimidade sexual6,8,12,14-17,19,21. As justificativas para as modificações na relação conjugal se dividem entre problemas físicos relacionados à progressão da doença ${ }^{14,19} \mathrm{e}$ aspectos psicológicos decorrentes da sobrecarga de cuidados, como a perda de intimidade ${ }^{12,14,16,20}$. Problemas de ereção por causa psicológica ${ }^{6}$ e perda de desejo sexual em mulheres também são relatados por pacientes e cônjuges como fatores psicológicos associados à insatisfação sexual7,8. Ainda, a perda de controle sobre o próprio corpo causada pela doença - incontinência e higiene pessoal pobre - foi considerada como fator físico importante, pois se refere à diminuição da intimidade e do interesse sexual. A pessoa com demência deixa de ser "sexy", o que lhe impossibilita ser desejada pelo parceiro6,14. 
Tabela 2. Satisfação sexual em idosos demenciados

\begin{tabular}{|c|c|c|c|c|}
\hline Autor/Ano & Amostra & Desenho & Instrumentos & Resultados \\
\hline Davies et al., 201019 & $\begin{array}{c}14 \text { cônjuges-cuidadores de pacientes com } \\
\text { demência e } 9 \text { cônjuges-cuidadores de } \\
\text { pacientes com CCL }\end{array}$ & $\begin{array}{c}\text { Transversal } \\
\text { não randomizado }\end{array}$ & $\begin{array}{c}\text { Questionários sobre intimidade e } \\
\text { sexualidade }\end{array}$ & $\begin{array}{l}\text { Diminuição da intimidade e sexualidade } \\
\text { devido à perda de memória do paciente }\end{array}$ \\
\hline Dourado et al., 2010 & $\begin{array}{l}15 \text { pacientes com demência leve e seus } \\
\text { cônjuges-cuidadores } \\
21 \text { pacientes com demência moderada e } \\
\text { seus cônjuges-cuidadores }\end{array}$ & $\begin{array}{c}\text { Transversal } \\
\text { não randomizado }\end{array}$ & $\begin{array}{l}\text { MMSE, CDR, ASPID, Cornell, } \\
\text { OSES, ZBI, entrevistas } \\
\text { semiestruturadas qualitativas }\end{array}$ & $\begin{array}{c}\text { Atividade e satisfação sexual são } \\
\text { influenciadas negativamente pelo avanço } \\
\text { da demência }\end{array}$ \\
\hline Hayes et al., 200914 & $\begin{array}{l}13 \text { cônjuges-cuidadores do sexo masculino } \\
15 \text { cônjuges-cuidadores do sexo feminino }\end{array}$ & $\begin{array}{c}\text { Qualitativo } \\
\text { não randomizado }\end{array}$ & Entrevistas qualitativas & $\begin{array}{l}\text { Perda de intimidade é causada por } \\
\text { mudanças decorrentes da demência, } \\
\text { resultando em sobrecarga do cuidador e } \\
\text { alteração da relação conjugal }\end{array}$ \\
\hline $\begin{array}{l}\text { Simonelli et al., } \\
2008^{12}\end{array}$ & $\begin{array}{c}100 \text { cônjuges-cuidadores em grupo de } \\
\text { intervenção } \\
100 \text { cônjuges de idosos sadios }\end{array}$ & $\begin{array}{l}\text { Caso controle } \\
\text { randomizado }\end{array}$ & $\begin{array}{c}\text { Entrevistas semiestruturadas, } \\
\text { CBI (T/dep-B, Dev-B, Phys-B, } \\
\text { Soc-B e Emot-B) }\end{array}$ & $\begin{array}{l}\text { Quanto maior a sobrecarga do cuidador, } \\
\text { menor a atividade sexual do casal }\end{array}$ \\
\hline Svetlik et al., 200520 & 136 cônjuges-cuidadores & $\begin{array}{c}\text { Transversal } \\
\text { não randomizado }\end{array}$ & ADL, NCSE, Questionários & $\begin{array}{l}\text { Quanto maior o cuidado com o paciente, } \\
\text { menor a satisfação sexual e intimidade }\end{array}$ \\
\hline Vugt et al., $2003^{17}$ & 53 cônjuges-cuidadores & $\begin{array}{c}\text { Transversal } \\
\text { não randomizado }\end{array}$ & Questionários, entrevistas, NPI & $\begin{array}{l}\text { Diminuição da intimidade sexual é } \\
\text { associada ao afastamento do paciente }\end{array}$ \\
\hline $\begin{array}{l}\text { Eloniemi-Sulkava et } \\
\text { al., 200215 }\end{array}$ & 42 cônjuges-cuidadores & $\begin{array}{c}\text { Transversal } \\
\text { não randomizado }\end{array}$ & $\begin{array}{c}\text { Entrevistas semiestruturadas por } \\
\text { telefone }\end{array}$ & $\begin{array}{l}\text { Há declínio no desejo sexual do paciente } \\
\text { com a progressão da doença }\end{array}$ \\
\hline Murray et al., $1998^{16}$ & 20 cônjuges-cuidadores & $\begin{array}{l}\text { Qualitativo } \\
\text { não randomizado }\end{array}$ & Entrevistas qualitativas & $\begin{array}{l}\text { Insatisfação nos cônjuges é gerada pelo } \\
\text { aumento de cuidados e pela perda de } \\
\text { intimidade }\end{array}$ \\
\hline Wright et al., 199813 & $\begin{array}{l}30 \text { pacientes com DA e seus cônjuges- } \\
\text { cuidadores em grupo de intervenção } \\
17 \text { casais de idosos sadios }\end{array}$ & $\begin{array}{l}\text { Caso controle } \\
\text { randomizado }\end{array}$ & MMSE, DAS, Questionários & $\begin{array}{l}\text { Demanda de pacientes por contato sexual } \\
\text { diminui com o avanço da doença }\end{array}$ \\
\hline Ballard et al., 199718 & $\begin{array}{l}40 \text { cônjuges-cuidadores de pacientes com } \\
\text { demência leve a moderada }\end{array}$ & $\begin{array}{c}\text { Transversal } \\
\text { não randomizado }\end{array}$ & $\begin{array}{l}\text { GMS, HAS, Cornell, BPSCSS, } \\
\text { SDS, MIS, CAMCOG, BSC }\end{array}$ & $\begin{array}{c}\text { Cuidadores que mantinham relação sexual } \\
\text { estavam satisfeitos e acreditavam que } \\
\text { seus parceiros também }\end{array}$ \\
\hline Wright et al., 199121 & $\begin{array}{l}60 \text { cônjuges-cuidadores em grupo de } \\
\text { intervenção } \\
34 \text { cônjuges de idosos sadios }\end{array}$ & $\begin{array}{l}\text { Grupo de intervenção } \\
\text { não randomizado; } \\
\text { grupo controle } \\
\text { randomizado }\end{array}$ & $\begin{array}{l}\text { Entrevistas semiestruturadas, } \\
\text { MMSE, GDS }\end{array}$ & $\begin{array}{l}\text { A qualidade sexual e afetiva no grupo com } \\
\text { DA declinou, comparada ao grupo saudável }\end{array}$ \\
\hline Litz et al., 19906 & 1 cônjuge-cuidador & Estudo de caso & Não informado & $\begin{array}{l}\text { Atividade sexual não é importante } \\
\text { Cuidador evita interesse sexual, pois } \\
\text { acredita estar se aproveitando de seu } \\
\text { parceiro }\end{array}$ \\
\hline
\end{tabular}

DA: Doença de Alzheimer; ADL: Activities of Daily Living; ASPID: Assessment Scale of Psychosocial Impact of Dementia; BPSCSS: Behaviour Problem Scale of the Carers Stress Scale; BSC: Burns Symptom Checklist; CAMCOG: Cambridge Cognitive Examination; CBI: Caregiver Burden Inventory (Dev-B: Development Burden; Emot-B: Emotional Burden; Phys-B: Physical Burden; Soc-B: Social Burden; T/dep-B: Time-dependent Burden); CCL: Comprometimento Cognitivo Leve; CDR: Clinical Dementia Rating; Cornell: Cornell Scale for Depression in Dementia; DAS: Dyadic Adjustment Rating Scale; GDS: Global Deterioration Scale; GMS: Geriatric Mental State Schedule; HAS: History and Aetiology Schedule; MIS: Marital Intimacy Scale; MMSE: Mini Mental State Examination; NCSE: Neurobehavioral Cognitive Status Examination; NPI: NeuroPsychiatic Inventory; QSES: Questionnaire on Sexual Experience and Satisfaction; ZBI: Zarit Burden Interview.

Os casais que mantêm relação sexual após o início da doença são significativamente mais jovens e casados há mais tempo do que casais que não a mantêm ${ }^{15}$.

Apenas um dos estudos relata que os déficits cognitivos resultantes do desenvolvimento da doença não acarretam em uma alteração na relação do casal, já que o cuidador tende a tolerar o declínio de seu cônjuge e dar suporte ${ }^{16}$. Vugt et al. ${ }^{17}$ descrevem que a deterioração do relacionamento conjugal pode estar mais especificamente associada aos sintomas de humor/apatia do paciente do que ao comprometimento cognitivo. Outras causas associadas à doença relacionam-se aos pacientes que se tornam mais egocêntricos e inflexíveis, além de diminuição da expressão verbal e alterações na personalidade (aumento de rigidez, apatia e egocentrismo, e controle emocional debilitado) ${ }^{19}$. Além disso, inúmeras mudanças negativas podem ocorrer no comportamento sexual do paciente, tornando-o incapaz de prestar atenção aos sentimentos e às necessidades sexuais de seu parceiro ${ }^{15}$. Nos estágios mais avançados, há dificuldade em manter a concentração na atividade sexual e esquecimento de relações sexuais anteriores ocorridas no mesmo dia, o que resulta em incômodo aos cônjuges e frequência exacerbada da atividade sexual6,21.

Quando a relação conjugal antes do diagnóstico é disfuncional, a interferência da doença parece ser maior ${ }^{8,16,19}$. Sendo assim, problemas de comunicação já existentes entre os parceiros podem ser observados, principalmente, nos estágios iniciais da doença ${ }^{16,19}$.
Os casais que mantinham uma relação estável e funcional antes do diagnóstico relataram significativa mudança nas expressões afetivas com o início do comprometimento de memória do cônjuge. Isso pode estar relacionado à perda do poder de decisão por parte dos pacientes e à mudança de papéis, na qual o cônjuge saudável assume uma função parental. Os cuidados diários com o paciente transformam o relacionamento do casal em uma relação de "pai e filho". Essa mudança afeta negativamente as dimensões emocional e sexual do casal ${ }^{19}$, além de trazer efeitos negativos na saúde do parceiro sexual ${ }^{20}$. Assim sendo, a saúde física e emocional do paciente e do cônjuge está relacionada diretamente com a atividade sexual do casal $8,13,15,19,21$.

Os estudos que incluem a visão dos pacientes sugerem que existe discordância entre os relatos de pacientes e cônjuges sobre a satisfação sexual do casal ${ }^{8,21}$. Os pacientes possuem dificuldades em reconhecer os diversos déficits cognitivos e funcionais. Dessa forma, o casal discorda sobre os motivos da interrupção ou da insatisfação sexual. A visão do paciente parece ser influenciada pelo comprometimento cognitivo, na medida em que a atividade sexual do passado pode ser relatada como sendo do presente. Entretanto, apesar da presença do comprometimento cognitivo, os diversos estudos sobre as divergências entre idosos com demência e seus parceiros atestam a validade da descrição dos pacientes sobre seu próprio estado e sua qualidade de vida ${ }^{8}$. Portanto, a inclusão dos relatos dos pacientes sobre sua satisfação sexual pode contribuir 
para um melhor entendimento e avaliação dessas discrepâncias, bem como sobre o bem-estar e a qualidade de vida do casal.

Um importante aspecto da sexualidade na demência está relacionado à substituição da atividade sexual por outros modos de demonstração de intimidade física, como abraços, beijos e toques ${ }^{6,19}$. Sendo assim, os relatos dos cônjuges saudáveis sobre a intimidade conjugal estão, também, relacionados à perda de qualidade da relação após o início da doença e ao progressivo comprometimento demencial ${ }^{14}$. Svetlik et al..$^{20}$ sugerem que o cônjuge resiliente que mantém o desejo sexual encontra satisfação de suas necessidades no afeto físico e na intimidade sexual de forma que o relacionamento é visto como menos angustiante. Quando há reciprocidade e intimidade no relacionamento do casal, o cuidado prestado pelo cônjuge é de melhor qualidade. Quando há queda nesses aspectos, o cuidado é insatisfatório e há um risco de doenças mentais no cuidador ${ }^{16}$. Parceiros saudáveis ficam relutantes em expressar suas dificuldades sexuais e também em procurar ajuda, pois acreditam que são egoístas, que estão se aproveitando do parceiro demenciado ${ }^{8}$ ou que o sexo perde prioridade 6 .

Existem diferenças entre satisfação sexual e gênero dos parceiros sexuais. A maior parte dos estudos apresenta cônjuges do sexo feminino $8,12-21$, que relatam ser difícil ter desejo e tempo para atividade sexual diante dos cuidados diários com o paciente. Ocorre um conflito entre as funções de cuidadora, mãe, amante e esposa, resultando em falta de interesse sexual14. A sobrecarga é maior em esposas cuidadoras, já que normalmente exercem esse papel sozinhas $8,16,19$. Cônjuges masculinos costumam receber ajuda nos cuidados com sua parceira, facilitando a preservação de sua posição de marido ${ }^{8,12}$. Cônjuges cuidadoras percebem mudanças em seus parceiros antes, se comparadas a cuidadores do sexo masculino e, por isso, os homens mantêm a atividade sexual por um período maior que as mulheres. Desse modo, cuidadores masculinos continuam considerando suas esposas como parceiras sexuais, já que os papéis conjugais estão resguardados, o que possibilita a manutenção das relações sexuais ${ }^{14}$.

Homens e mulheres entendem intimidade e cuidados de forma diferenciada ${ }^{14}$. Cônjuges do sexo masculino que mantêm a prática sexual com sua parceira demenciada se mostram mais interessados na manutenção dessa relação, mas se afastam quando sentem que sua esposa não está demonstrando prazer ou interesse $e^{6,18}$. Esses cônjuges relatam sentimentos de estar se aproveitando de alguém que não tem mais o poder de decisão, pois, para eles, não é essencial que a parceira os reconheça, desde que ela seja capaz de consentir com o ato sexual14. Por outro lado, para as esposas, a incapacidade de reconhecimento é um dos principais motivos para a falta de atividade sexual ${ }^{6}$. Entretanto, há estudos em que o gênero não foi considerado como fator significativo para as mudanças na atividade sexual $8,15,20$.

A frequência da atividade sexual declina com o avanço do comprometimento causado pela demência ${ }^{13}$. Cônjuges percebem que, para o paciente, a relação sexual se torna menos importante após o início da doença ${ }^{15}$. Os cônjuges mais insatisfeitos com a ausência de atividade sexual estão associados aos pacientes mais jovens e com a linguagem expressiva preservada ${ }^{18}$.

Os cuidados com o paciente nem sempre trazem apenas experiências negativas para o casal. Existem situações nas quais os cônjuges saudáveis podem ser mais afetuosos e aproveitar melhor o tempo juntos depois do início da doença. As dificuldades vivenciadas pelo paciente com o avanço da demência despertam sentimentos de empatia por parte do parceiro, que deseja protegê-lo ${ }^{17}$. Alguns pacientes apresentam até mesmo mudanças positivas no comportamento sexual, após diagnosticada a doença ${ }^{15}$.

\section{Conclusão}

A intimidade e a atividade sexual na demência são influenciadas negativamente pela relação de cuidados decorrente da doença. A sobrecarga de cuidados e a alteração de papéis na relação conjugal foram consideradas as principais causas para o declínio da atividade sexual. A disfunção erétil em pacientes e cônjuges, a capacidade decisória para o consentimento da relação sexual por parte do paciente demenciado e os problemas referentes à idade e à saúde (física e emocional) do cônjuge e/ou paciente foram os fatores associados à insatisfação sexual.

Por outro lado, a intimidade pode ser influenciada positivamente pela demência, por meio de demonstrações de carinho e empatia entre os cônjuges e não necessariamente pela atividade sexual.

Os achados dos poucos estudos na área indicam que a atividade sexual na demência é um campo promissor de pesquisa. Assim, é necessário aumentar a representatividade desses resultados por meio de pesquisas metodologicamente bem desenhadas, de modo a melhor investigar a modificação na atividade, no comportamento e na expectativa relacionados à satisfação/insatisfação sexual desses casais.

\section{Referências}

1. Langer N. Late Life Love and Intimacy. Educational Gerontology. 2009;35:752-64.

2. Vasconcellos D, Novo R, Castro O, Vion-Dury K, Ruschel A, Couto M, et al. A sexualidade no processo do envelhecimento: novas perspectivas - comparação transcultural. Estud Psicol. 2004;9(3):413-9.

3. DSM-IV-TR. Manual diagnóstico e estatístico de desordens mentais. Trad. Cláudia Dornelles. 4.ed. Porto Alegre: Artmed; 2002.

4. Graham, N, Lindesay J, Katona C, Bertolote J, Camus V, Copeland J, et al. Redução da estigmatização e da discriminação das pessoas idosas com transtornos mentais: uma declaração técnica de consenso. Rev Psiq Clín. 2007;34(1):39-49.

5. Derouesné C. The so-called hypersexual behaviors in dementia. Psychol Neuropsychiatr Vieil. 2009;7(2):101-8.

6. Litz BT, Zeiss AM, Davies HD. Sexual concerns of male spouses of female Alzheimer's disease patients. Gerontologist. 1990;30(1):113-6.

7. Davies HD, Zeiss A, Tinklenberg JR. 'Til Death Do Us Part: intimacy and sexuality in the marriages of Alzheimer's patients. J Psychosoc Nurs Ment Health Services. 1992;30(11):6-10.

8. Dourado M, Finamore C, Barroso MF, Santos R, Laks J. Sexual satisfaction in dementia: perspectives of patients and spouses. Sex Disabil. 2010; 28:195-203.

9. Sousa MFB, Santos RL, Arcoverde C, Dourado M, Laks J. Consciência da doença na doença de Alzheimer: resultados preliminares de um estudo longitudinal. Rev Psiq Clin. 2011;38(2):57-60.

10. Dourado M, Laks J, Leibing A, Engelhardt E. Consciência da doença na demência. Rev Psiq Clin. 2006;33(6):313-21.

11. Szwabo PA. Counseling about sexuality in the older person. Clin Geriatr Med. 2003;19(3):595-604.

12. Simonelli C, Tripodi F, Rossi R, Fabrizi A, Lembo D, Cosmi V, et al. The influence of caregiver burden on sexual intimacy and marital satisfaction in couples with an Alzheimer spouse. Int J Clin Pract. 2008;62(1):47-52.

13. Wright LK. Affection and sexuality in the presence of Alzheimer's disease: a longitudinal study. Sex Disabil. 1998;16(3):167-79.

14. Hayes J, Boylstein C, Zimmerman MK. Livind and loving with dementia: negotiating spousal and caregiver identity through narrative. J Aging Stud. 2009;23(1):48-59.

15. Eloniemi-Sulkava U, Notkola I, Hämäläinen K, Rahkonen T, Virammo P, Hentinen M, et al. Spouse caregivers' perceptions of influence of dementia on marriage. Int Psychogeriatr. 2002;14(1):47-58.

16. Murray J, Livingston G. A qualitative study of adjustment to caring for an older spouse with psychiatric illness. Ageing Soc. 1998;18:659-71.

17. Vugt ME, Stevens F, Aalten P, Lousberg R, Jaspers N, Winkens I, et al. Behavioural disturbances in dementia patients and quality of the marital relationship. Int J Geriatr Psychiatry. 2003;18:149-54.

18. Ballard CG, Solis M, Gahir M, Cullen P, George S, Oyebode F, et al. Sexual relationship in married dementia sufferers. Int J Geriatr Psychiatry. 1997;12:447-51.

19. Davies HD, Newkirk LA, Pitts CB, Coughlin CA, Sridhar SB, Zeiss LM, et al. The impact of dementia and mild memory impairment (MMI) on intimacy and sexuality in spousal relationships. Int Psychogeriatr. 2010;22:618-28.

20. Svetlik D, Dooley WK, Weiner MF, Williamson GM, Walters AS. Declines in satisfaction with physical intimacy predict caregiver perceptions of overall relationship loss: a study of eldery caregiving spousal dyads. Sex Disabil. 2005;23(2):65-79.

21. Wright LK. The impact of Alzheimer's disease on the marital relationship. Gerontologist. 1991;31:224-37. 